\title{
Analyse spectrale singulière des signaux vibratoires et Machine Learning pour la surveillance d'usure d'outils
}

\author{
Bovic Kilundu a et Pierre Dehombreux \\ Service de génie mécanique, Faculté Polytechnique de Mons, 53 rue du Joncquois, 7000 Mons, Belgique
}

Reçu le 19 novembre 2006, accepté le 25 mai 2007

\begin{abstract}
Résumé - Cette étude explore l'utilisation des techniques de Machine Learning pour la classification de l'état d'outils en usinage. Une analyse spectrale singulière (ASS) pseudo-locale des signaux vibratoires relevés sur le porte-outil, couplée à un filtrage passe-bande a permis la définition et la mise en évidence d'indicateurs très sensibles à l'évolution de l'état de l'outil. Ces indicateurs sont définis à partir des sommes des raies spectrales des signaux reconstruits par ASS et de leurs résidus, dans des gammes de fréquence judicieusement choisies. Les taux de reconnaissance de l'usure sont très bons et dépassent les $80 \%$. Cette étude met en évidence deux aspects importants : la forte richesse en information des composantes hautes fréquences des signaux vibratoires et la possibilité de s'affranchir du bruit inutile par la combinaison de l'ASS et d'un filtrage passe-bande.
\end{abstract}

Mots clés : Machine Learning / signaux vibratoires / analyse spectrale singulière / surveillance de l'usure d'outils

\begin{abstract}
Singular spectrum analysis and Machine Learning techniques for tool wear monitoring. This study explores the use of Machine Learning techniques for tool condition monitoring in metal machining. Pseudo-local Singular Spectrum Analysis (SSA) of vibration signals raised on the tool holder, coupled to a pass-band filter allowed the definition and the extraction of features which are very sensitive to the tool wear. These features are defined from sums of Fourier coefficients of the signals reconstructed by SSA and of their residues, in judiciously selected frequency bands. The rates of recognition of wear are very good and exceed $80 \%$. This study highlights two important aspects: strong relevance of information in high frequency vibration components and benefits of the combination of SSA and pass-band filtering to get rid of useless components (noise).
\end{abstract}

Key words: Machine Learning / vibration signal / singular spectrum analysis / tool condition monitoring

\section{Introduction}

La minimisation des coûts se présente comme une exigence incontournable dans tous les secteurs de production, car avec la mondialisation de l'économie seuls les produits réalisés à un coût raisonnable peuvent encore trouver leur place sur les marchés. L'état de l'outil et sa durée de vie sont des composantes critiques du coût d'usinage. Il est donc important de pouvoir élaborer un moyen de détection d'usure et de prédiction de la durée de vie de l'outil pour une gestion rationnelle du temps de production. Les tolérances dimensionnelles et la qualité des pièces usinées en dépendent.

\footnotetext{
a Auteur pour correspondance :

bovic.kilundu@student.fpms.ac.be
}

Il ne sera pas question d'empêcher l'usure qui est un phénomène inhérent à tout processus de coupe vu les niveaux de contraintes, de frottement et de température auxquels l'outil est soumis. Mais une méthode permettant la détection de l'apparition de l'usure et de son évolution est un réel besoin dans le cadre d'une politique « juste à temps $»$ de changement d'outil $[1,2]$.

Les méthodes de surveillance d'outils sont généralement classées en deux groupes dont :

1. les méthodes directes où l'usure est directement mesurée en utilisant des capteurs optiques, radioactifs ou des capteurs basés sur la résistance électrique;

2. les méthodes indirectes qui procèdent par une évaluation de l'usure sur base des paramètres mesurés pendant le processus de coupe : l'effort de coupe, l'émission acoustique ou les vibrations. 
Toutes ces méthodes peuvent être classées en méthodes on-line appliquées pendant la coupe et en méthodes off-line où l'on interrompt le processus pour procéder au contrôle.

Le développement d'un système de surveillance passe par deux étapes principales, après le choix de la méthode, donc du type de capteur à utiliser. Il s'agit de la procédure d'extraction des indicateurs des signaux bruts mesurés, et de la classification et du développement d'une stratégie de décision [3]. Plusieurs études ont adressé des techniques de machine learning appliquées à la surveillance d'outils. Mais, comme dans tout problème de surveillance, le plus grand défi a toujours été la définition d'indicateurs qui puissent suivre fidèlement le phénomène surveillé et traduire les signes d'une avarie. Abu-Mahfouz [4] a extrait, pour la détection et la classification de l'état de l'outil en perçage à l'aide des réseaux de neurones, des indicateurs du domaine fréquentiel en procédant à la moyenne des coefficients d'une décomposition en ondelettes des vibrations et en considérant le maximum des pics du spectre d'entropie. Obikawa et al. [5] ont aussi fait usage des coefficients d'ondelettes des signaux d'effort de coupe.

Dans sa revue des méthodes indirectes de monitoring d'outils, Dimla $[6,7]$ cite plusieurs travaux qui ont analysé la corrélation entre l'état de l'outil et les vibrations. El-Wardany et al. [8] ont utilisé, dans le domaine temporel, le rapport entre le kurtosis et la valeur moyenne du signal vibratoire. Pour leur part, Jiang et al. [9] ont montré que l'énergie dans certaines bandes de fréquence judicieusement choisies était sensible à l'évolution de l'état de l'outil. Diverses méthodes simples d'extraction d'indicateurs sont aussi rapportées comme ayant fourni de bons résultats, c'est par exemple des valeurs moyennes dans des bandes de fréquence données [10] et quelques pics dans le spectre vibratoire [11]. Shi et Gindy [12] ont par contre trouvé une faible corrélation entre la valeur moyenne et l'écart type d'un signal avec l'état de l'outil, mais ils ont plutôt montré l'utilité d'une analyse en composantes principales pour l'extraction d'indicateurs quand deux capteurs sont utilisés suivant des directions perpendiculaires.

Cependant, la surveillance d'outil est une activité assez difficile à mettre en œuvre vu la non-stationarité du processus de coupe et l'impossibilité d'utiliser en pratique les algorithmes souvent lourds discutés dans de nombreux rapports de recherche. Garret [13] souligne aussi le haut niveau de bruit dans les signaux vibratoires et acoustiques relevés en vue de la surveillance d'outil comme une difficulté supplémentaire.

Dans cette étude, est développée une modélisation d'un système de surveillance de l'état de l'outil à l'aide des techniques de Machine Learning sur base des mesures vibratoires. Les variables utilisées pour la classification sont extraites des signaux par la méthode d'analyse spectrale singulière. Cette méthode d'analyse est principalement dédiée au traitement des séries temporelles courtes et bruitées. Elle est très utilisée pour l'analyse des données géophysiques et climatiques [14]. Elle permet la décomposition du signal en plusieurs composantes, ce qui donne l'espoir de pouvoir isoler la composante utile ayant

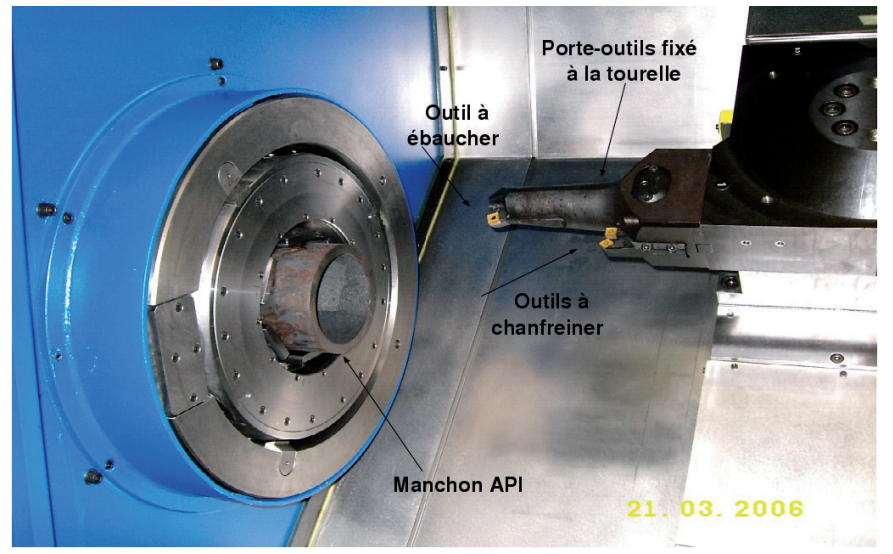

Fig. 1. Machine à fileter (avec la permission de M.P. Colinet sprl).

une relation directe avec l'évolution de l'outil. Salgado et Alonso [15] l'ont appliquée à la surveillance d'usure d'outil en tournage comme moyen d'extraction des indicateurs à fournir à un classifieur de type réseau de neurones. D'autres applications avec des réseaux de neurones ont été faites dans le domaine de surveillance des processus industriels $[16,17]$.

Combinée à un filtrage passe-bande, l'analyse spectrale singulière s'est révélée être un excellent moyen d'extraction d'indicateurs pour la reconnaissance de l'usure d'outil.

\section{Cadre de l'étude}

Cette étude est menée sur des machines à fileter les manchons de raccord des tubes de cuvelage selon les normes $\mathrm{API}^{1}$ (Fig. 1). Les accéléromètres sont fixés à la tourelle, du côté opposé au porte-outil. Trois capteurs monoaxiaux sont utilisés (Fig. 2) :

- le premier est orienté suivant la direction $x$, parallèle au porte-outil et à l'axe du manchon à usiner ;

- le second suivant la direction $y$ orthogonale à $x$;

- et le troisième suivant la direction $z$ perpendiculaire au plan de la tourelle et parallèle à l'effort de coupe.

Pour le dégrossissage comme pour le filetage l'usinage est fait en deux temps, d'abord d'une première extrémité vers le centre puis de l'autre vers le centre après indexation du mandrin.

Lors du dégrossissage, l'usinage est conique c'est-àdire la quantité de matière enlevée n'est pas la même en toute position de l'outil suivant l'axe du manchon. Cette quantité de matière enlevée est dégressive de l'extrémité du manchon vers le milieu suivant une conicité de 6,25\% (Fig. 3). Les manchons usinés sont en acier de nuance API L80.

Les signaux vibratoires relevés à l'aide d'un système d'acquisition multi-canaux ont les caractéristiques suivantes :

- fréquence d'échantillonnage : $51,2 \mathrm{kHz}$, pour une gamme d'analyse de $0-20 \mathrm{kHz}$;

\footnotetext{
1 American Petroleum Institute.
} 


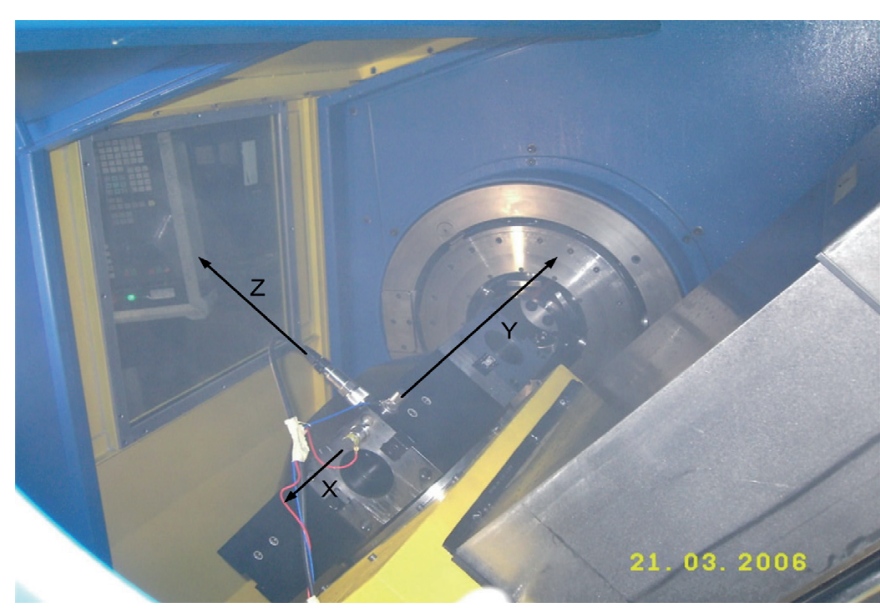

Fig. 2. Orientation des capteurs.

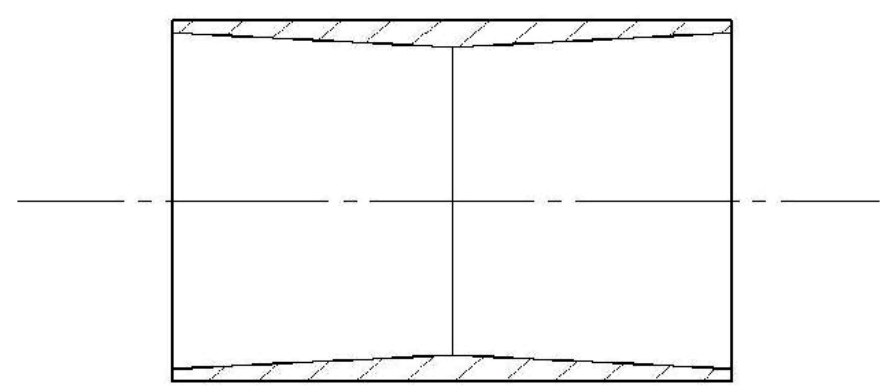

Fig. 3. Coupe de l'ébauche.

Tableau 1. État de l'outil et du processus de coupe pour les 43 essais.

\begin{tabular}{lcc}
\hline État & Symbole & Effectif \\
\hline Plaquette neuve ou comme neuve & $\mathrm{N}$ & 22 \\
Faible usure & $\mathrm{U} 1$ & 7 \\
Usure assez avancée & $\mathrm{U} 2$ & 6 \\
Bourrage copeau & $\mathrm{BC}$ & 4 \\
Passage à vide & $\mathrm{V}$ & 4 \\
\hline
\end{tabular}

- taille échantillon : variable en fonction de la durée de l'opération d'usinage (en moyenne $20 \mathrm{~s}$ ).

Cette campagne de mesures a été effectuée à l'occasion des essais de mise en marche de la machine. L'étude a été menée sur un ensemble de 43 signaux représentant principalement 5 états de fonctionnement dont trois caractérisent l'état de l'outil (N, U1, U2) et deux le processus (BC, V) comme repris dans le tableau 1.

L'état de l'outil a été caractérisé qualitativement sans quantification de l'usure. On a distingué un outil bon de celui usé faiblement ou de manière avancée.

Cette étude va se focaliser sur le processus d'ébauche et ne considérer que le seul signal relevé suivant la direction $Z$ pour la simple raison que l'accélération mesurée y est la plus grande. C'est la direction tangentielle, donc parallèle à l'effort de coupe.

\section{Analyse Spectrale Singulière (ASS)}

L'ASS est une technique non-paramétrique d'analyse de séries temporelles basée sur les principes de statistiques multivariées. Elle permet la décomposition du signal de départ en signaux indépendants, mais dont la somme donne le signal de départ. Ces composantes indépendantes sont souvent construites de manière à distinguer une composante donnant la tendance, et d'autres représentant respectivement le contenu oscillatoire et le bruit $[18,19]$.

Le point de départ de l'ASS est la construction d'une matrice dite « de trajectoire » qui est composée de vecteurs de dimension $M$ extraits de le série temporelle.

Soit $\{X(t) \mid t=1, \ldots, N\}$ une série temporelle de longueur $N$. La séquence des vecteurs $\widetilde{X}(t)$ de dimension $M$ est obtenue en parcourant, avec recouvrement, la série de données à l'aide d'une fenêtre glissante de longueur $M$. On obtient ainsi

$$
\tilde{X}(t)=(X(t), X(t+1), \ldots, X(t+M-1))
$$

les vecteurs $\tilde{X}(t)$ sont indexés par $t=1, \ldots, N^{\prime}$, où $N^{\prime}=$ $N-M+1$. Ils vont constituer les colonnes de la matrice de trajectoire $T=\left(\tilde{X}_{1}, \tilde{X}_{2}, \cdots, \tilde{X}_{N^{\prime}}\right)$,

$$
T=\left(\begin{array}{cccc}
X(1) & X(2) & \cdots & X(N-M+1) \\
X(2) & X(3) & \cdots & X(N-M+2) \\
\vdots & \vdots & \ddots & \vdots \\
X(M-1) & X(M) & \cdots & X(N-1) \\
X(M) & X(M+1) & \cdots & X(N)
\end{array}\right)
$$

Cette matrice ainsi construite est une matrice de Hankel, c'est-à-dire les éléments des diagonales montantes sont identiques. Le choix de la longueur de la fenêtre est un compromis entre deux considérations : la quantité d'information extraite et le degré de confiance statistique en cette information. Le premier aspect requiert une fenêtre aussi large que possible alors que le second aspect exige plusieurs répétitions de fenêtre, c'est-à-dire un rapport $N / M$ aussi grand que possible $[14,18]$.

On procède, ensuite, à une projection de la série temporelle dans l'espace des vecteurs propres de la matrice de covariance de $T$. Cette projection donne les composantes principales $A_{k}$.

$$
A_{k}(t)=\sum_{j=1}^{M} X(t+j-1) \rho_{k}(j)
$$

où $\rho_{k}$ représente les vecteurs propres de la matrice de covariance de $T$.

On peut alors reconstruire la partie de la série temporelle associée à un seul ou à plusieurs vecteurs propres en combinant les composantes principales y associées :

$$
y_{k}(t)=\frac{1}{M_{t}} \sum_{k \in D} \sum_{j=L_{t}}^{U_{t}} A_{k}(t-j+1) \rho_{k}(j)
$$

où $D$ représente les indices des composantes principales choisies pour la reconstruction. La valeur du facteur de 

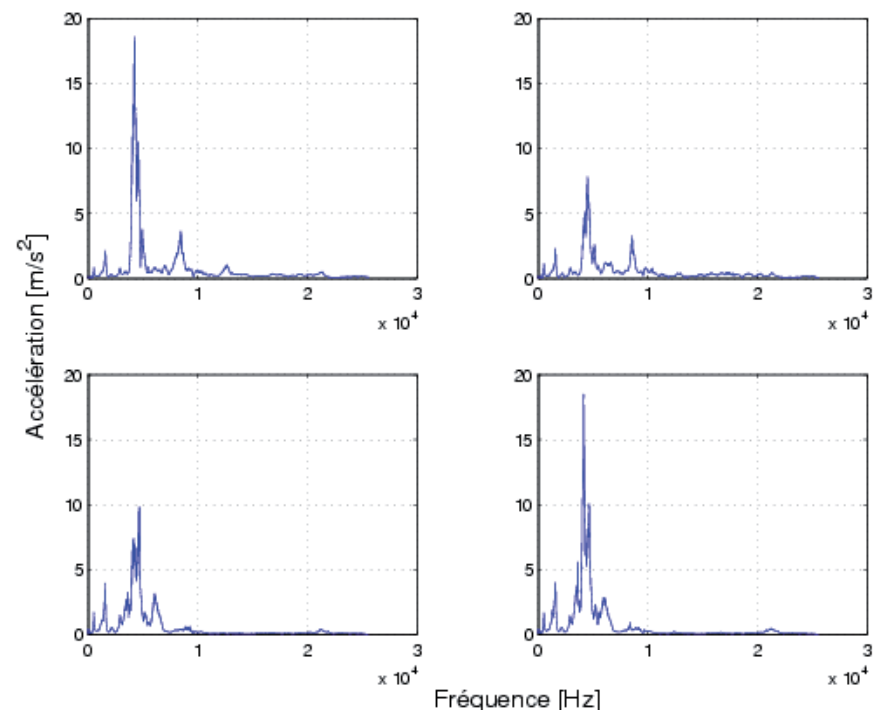

Fréquence $[\mathrm{Hz}]$

Fig. 4. Quelques spectres fréquentiels pour le processus d'ébauche.

normalisation $M_{t}$, et celles de $L_{t}$ et $U_{t}$ sont déterminées par l'algorithme de moyennage diagonal [19]

$\left(M_{t}, L_{t}, U_{t}\right)= \begin{cases}(t, 1, t), & 1 \leqslant t \leqslant M-1 \\ (M, 1, M), & M \leqslant t \leqslant N^{\prime} \\ (N-t+1, t-N+M, M), & N^{\prime}+1 \leqslant t \leqslant N\end{cases}$

Cette étude a concerné des signaux très longs représentant un cycle de coupe de durée moyenne $20 \mathrm{~s}$. L'échantillonnage ayant été réalisé à plus de $50 \mathrm{kHz}$, la longueur moyenne des signaux est de $10^{6}$ points. Pour tenir compte de la non-stationnarité du processus de coupe et afin de contourner la difficulté liée au temps de calcul lorsqu'on manipule de longs signaux, une technique similaire à l'ASS locale a été utilisée [20]. Elle a consisté à procéder localement à l'ASS sur des segments de signal de longueur $W$, avec $M=50<W=N / 50<N$. Ces fenêtres de longueurs $W$ sont définies sans recouvrement sur le signal d'origine. Le signal reconstruit est obtenu à partir des portions reconstruites localement.

\section{Extraction d'indicateurs}

La figure 4 montre que l'essentiel de l'information se trouve contenu dans les composantes fréquentielles endessous de $6000 \mathrm{~Hz}$. Ceci suggère un sous-échantillonnage par 4 du signal, mais avant cela le signal est filtré afin de prévenir l'aliasing. Cette diminution du nombre d'échantillons est effectuée essentiellement dans le but de réduire le temps de calcul lors des traitements subséquents.

Pour l'ASS, le signal original est d'abord découpé en 50 segments sur lesquels est effectuée l'ASS à l'aide d'une

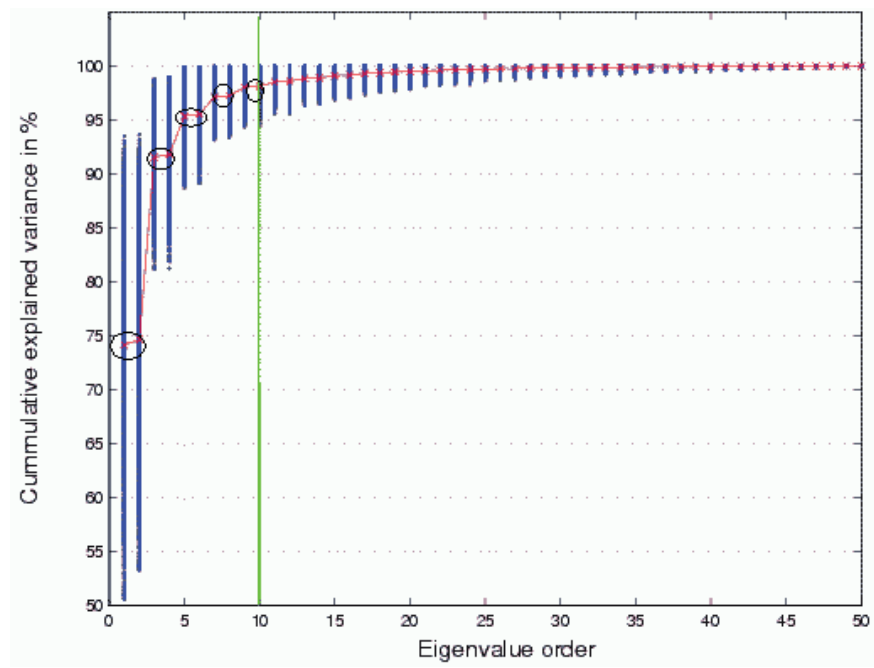

Fig. 5. Contribution moyenne à la variance totale pour chaque valeur propre.

fenêtre glissante de longueur 50. On peut remarquer sur la figure 5 que les 10 premières valeurs propres contribuent pour plus de $95 \%$ à la variance totale des données, les valeurs propres ayant été préalablement triées en ordre décroissant. Il s'ensuit que la reconstruction sera basée sur seulement les 10 premières composantes principales et ces composantes seront groupées 2 par 2 puisque la contribution moyenne à la variance totale est presque la même pour 2 valeurs propres successives. Nous obtenons 5 signaux reconstruits par signal original.

Pour chaque signal reconstruit, noté RC, on évalue aussi son résidu c'est-à-dire la somme des quatre autres signaux restants. L'objectif est de définir des indicateurs à partir de la notion de rapport signal/bruit, en considérant que le résidu représenterait le bruit si toute l'information utile du signal d'origine était contenue dans la composante reconstruite. Les figures 6 et 7 illustrent, pour un essai, le temporel et le spectre fréquentiel des signaux reconstruits et de leurs résidus pour le cas de RC1 et RC5. La reconstruction de RC1 est basée sur les deux premières des 10 composantes principales, alors que celle de RC5 est basée sur les deux dernières. On pourra remarquer que RC1 est riche en hautes fréquences à l'opposé de RC5. Les signaux reconstruits intermédiaires auront, eux bien sûr, un contenu intermédiaire entre celui de RC1 et celui de RC5.

Chaque signal reconstruit est filtré dans les 3 gammes de fréquence suivantes $10-2000 \mathrm{~Hz}, 2000-4000 \mathrm{~Hz}$ et 4000-6000 Hz à l'aide d'un filtre de Butterworth. Cette phase de traitement est proposée afin de construire des indicateurs selon les gammes de fréquence qui s'imposent par la configuration même du contenu spectral des signaux traités. En effet, en examinant les spectres fréquentiels des signaux relatifs aux différents processus d'ébauche, on remarquera à partir des quelques spectres représentés à la figure 4 que l'essentiel de l'énergie vibratoire est contenu dans la bande de fréquence $2-6 \mathrm{kHz}$. 

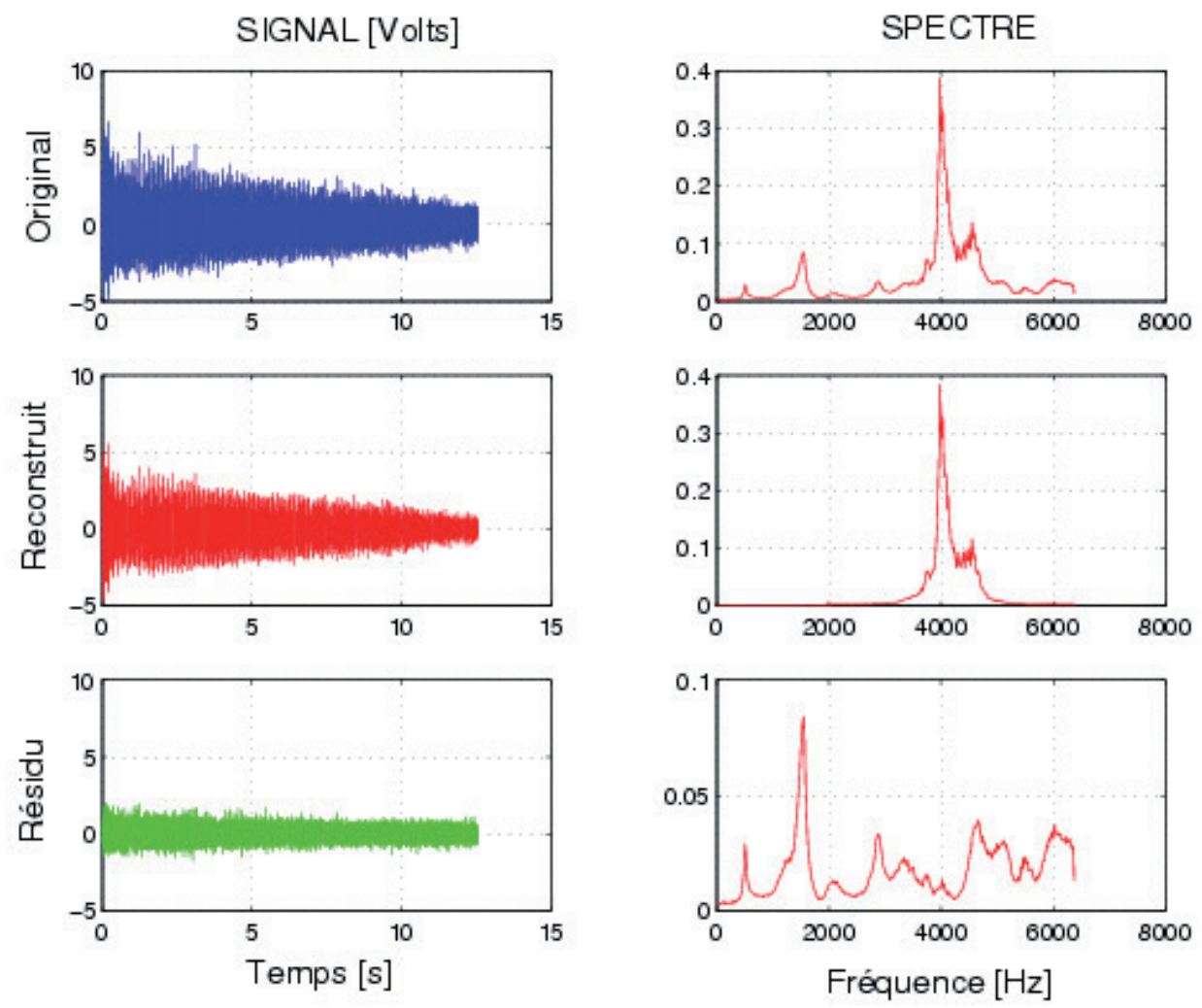

Fig. 6. Signaux et spectres relatifs à la composante RC1.
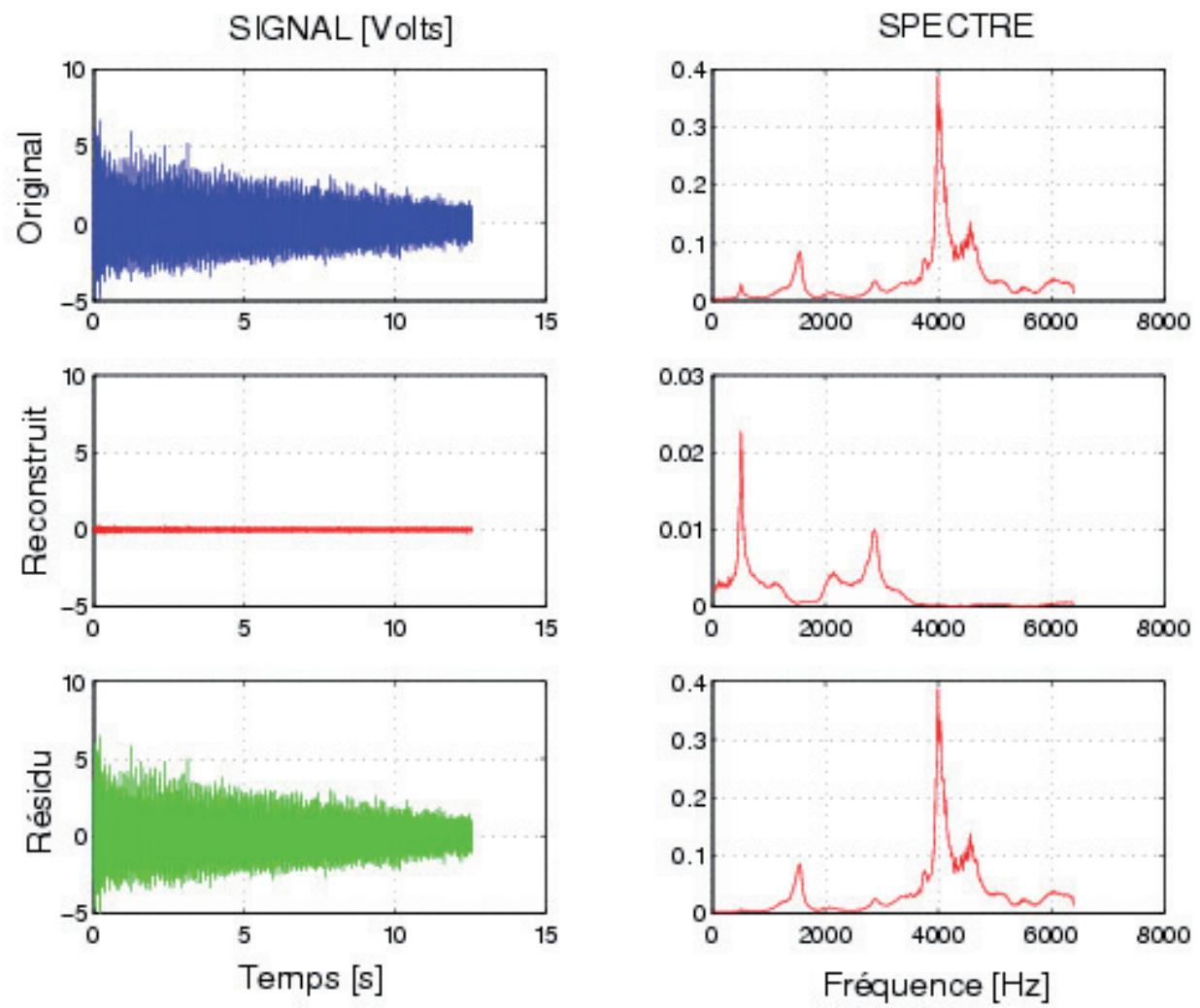

Fig. 7. Signaux et spectres relatifs à la composante RC5. 

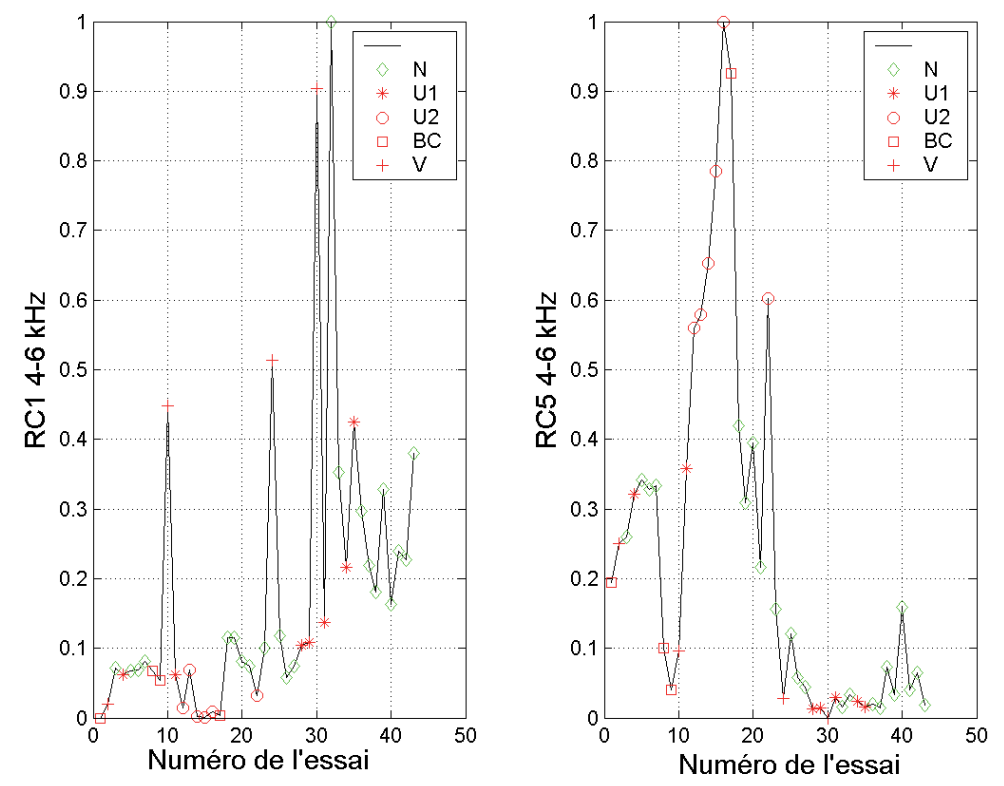

Fig. 8. Évolution des variables RC1 et RC5 4-6 kHz en fonction du numéro de l'essai.

Dans chaque gamme de fréquence, les indicateurs $v$ extraits dans le but de la classification sont calculés de la manière suivante :

- si $y_{n}$ représente la série du signal reconstruit et $r_{n}$ celle du résidu correspondant $(n=1 \ldots N)$,

$$
v=B \frac{\sum_{k}\left\|G_{k}^{r}\right\|}{\sum_{k}\left\|G_{k}^{y}\right\|}
$$

où $B$ un facteur de normalisation tenant compte de l'avance $A$ et de la vitesse de coupe $V$, et $G_{k}^{y}$ et $G_{k}^{r}$ respectivement les coefficients des transformées de Fourier de $y_{n}$ et $r_{n}$ sont obtenus comme suit :

$$
\begin{aligned}
& B=\frac{1}{A \cdot V} \\
& G_{k}^{y}=\frac{1}{N} \sum_{n=1}^{N} y_{n} e^{-j 2 \pi k n / N} \\
& G_{k}^{r}=\frac{1}{N} \sum_{n=1}^{N} r_{n} e^{-j 2 \pi k n / N}
\end{aligned}
$$

Ce qui aboutit à 15 variables pour chaque signal brut relevé sur le porte-outil ( 3 bandes fréquentielles pour chacune des 5 reconstructions du signal). Parmi ces 15 variables, certaines semblent suivre fidèlement l'évolution de l'état de l'outil et l'apparition de certains phénomènes tels que le bourrage copeau ou un passage presque à vide de l'outil. Sur la figure 8 qui représente les variables RC1 4000-6000 Hz et RC5 4000-6000 Hz normalisées pour les 43 essais, on remarquera que RC5 dans la gamme 4000$6000 \mathrm{~Hz}$ traduit l'évolution de l'usure de l'outil alors que les pics de RC1 dans la même gamme sont liés à un passage à vide. En effet, la classe N est caractérisée par de faibles valeurs de RC5 4000-6000 Hz tandis que U2 correspond à ses grandes valeurs.
Pour tenir compte d'une éventuelle dépendance de l'usure vis-à-vis des paramètres de coupe, à ces 15 variables sont ensuite ajoutées l'avance, la vitesse de coupe et le diamètre du manchon

\section{Résultats et discussion}

Quatre méthodes de classification ont été testées sur ce jeu de données :

- les réseaux bayésiens;

- les $k$ plus proches voisins (KNN);

- les arbres de décisions;

- et les réseaux de neurones.

Avant d'alimenter les classifieurs, les données sont réduites dans l'intervalle $[0,1]$ par une normalisation linéaire, puis une sélection de variables pertinentes pour le problème est effectuée. Il est établi que l'utilisation des indicateurs non pertinents peut sensiblement entamer les performances d'un classifieur (arbre de décision, réseaux de neurones, règles de classification, etc.) [21]. La sélection des variables se fait en considérant le sous-ensemble qui permet une meilleure séparation des classes.

Dans la recherche d'un sous-ensemble composé des variables pertinentes, il y a essentiellement deux approches différentes. La première procède à une évaluation basée sur les caractéristiques générales de données; tandis que la deuxième évalue les sous-ensembles en faisant usage d'un algorithme de classification. Cette sélection n'a retenu que 4 des 18 variables de départ :

- la variable RC1 4000-6000 Hz;

- la variable RC3 2000-4000 Hz;

- la variable RC5 4000-6000 Hz;

- l'avance A. 
Tableau 2. Taux de reconnaissance de l'état du processus (en \%).

\begin{tabular}{l|cc}
\hline & \multicolumn{2}{|c}{ Taux de classification } \\
\hline Méthode & Espace complet & Espace réduit \\
\hline Réseaux bayésiens & 53,5 & 55,8 \\
KNN & 67,4 & 76,7 \\
Arbres de décisions & 62,8 & 76,7 \\
Réseaux de neurones & 72,9 & 76,7 \\
\hline
\end{tabular}

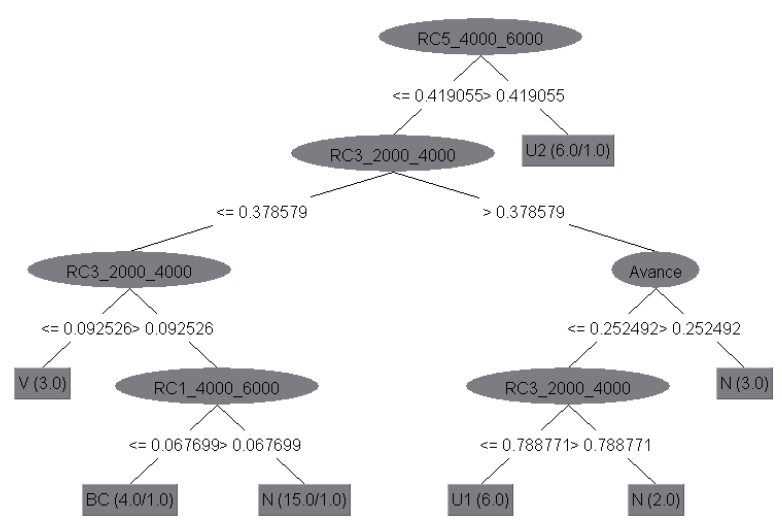

Fig. 9. Arbre de décision.

Comme le jeu de données n'est pas large, afin d'améliorer le processus de classification les ensembles d'apprentissage et de test ont été construits sur base de l'algorithme de validation croisée à $k$ blocs $(k$-fold cross validation) [22]. Cet algorithme consiste à créer, dans l'ensemble de données $D, k$ sous-ensembles (ici $k=10$ blocs) $D_{i}$ disjoints tels que leur union donne l'ensemble de données. Chaque bloc $D_{i}$ est ensuite utilisé comme ensemble d'apprentissage et le test effectué sur le complément à $D$ de $D_{i}$. À la fin du processus l'erreur de généralisation est estimée en faisant la moyenne des erreurs obtenues pour chaque bloc.

Ce processus de classification a été mené dans l'environnement « Weka KnowledgeFlow » [21]. Le tableau 2 donne les résultats globaux de classification. On y reprend le taux de classification sur l'ensemble complet des variables à côté de celui obtenu sur l'espace réduit ne contenant que les variables jugées pertinentes à l'issue de la sélection. La figure 9 illustre l'arbre de décision construit sur ces données.

On aura observé une effective amélioration des résultats de classification dans le cas de l'espace réduit. Un autre bénéfice est la réduction du temps de calcul pour le processus de classification qui ne concerne plus que 4 variables au lieu de 18 .

L'examen des détails du tableau 3 révèle de très bons résultats de reconnaissance de l'état de l'outil pour presque toutes les méthodes sauf pour le réseaux bayésiens qui ont mal reconnu beaucoup d'instances U1 en les classifiant comme des N. Le taux de reconnaissance des classes relatives à l'état du processus (BC et $\mathrm{V}$ ) est très faible. Particulièrement le passage à vide a été
Tableau 3. Taux de reconnaissance de l'état de l'outil (en \%).

\begin{tabular}{llllll}
\hline & $\mathrm{N}$ & $\mathrm{U} 1$ & $\mathrm{U} 2$ & $\mathrm{BC}$ & $\mathrm{V}$ \\
\hline Réseaux bayésiens & 72,7 & 42,9 & 66,7 & 25,0 & 0,0 \\
KNN & 90,9 & 85,7 & 83,3 & 50,0 & 0,0 \\
Arbres de décisions & 72,7 & 100 & 100 & 50,0 & 50,0 \\
Réseaux de neurones & 95,5 & 100 & 83,3 & 0,0 & 0,0 \\
\hline
\end{tabular}

classifié comme N, ce qui pourrait s'expliquer par le faible niveau de contrainte qui est naturellement, et à une certaine échelle, rencontré dans le cas d'un outil neuf. En ce qui concerne la reconnaissance du bourrage de copeaux, les matrices de confusion relatives aux différentes classifieurs ont révélé que $\mathrm{BC}$ a été classifié comme $\mathrm{N}$ ou comme U2. Une explication à cela est évidente du fait que la condition BC ne caractérise pas l'état de l'outil. En effet, un bourrage de copeaux peut s'être produit lors d'une coupe avec un outil neuf ou usé. Cette observation a revélé la robustesse des indicateurs vis-à-vis du bruit issu du fouettement de copeaux lors du bourrage.

\section{Conclusion}

La surveillance de l'usure d'outils par des méthodes indirectes demeure un champ d'investigation ouvert, particulièrement pour la détermination du type de signal à utiliser et le traitement à lui imposer pour l'extraction d'indicateurs. Cette étude ne s'est pas seulement intéressée à l'état de l'outil, mais aussi à d'autres dysfonctionnements du processus tels que le bourrage de copeau ou un passage à vide. Elle a exploré une méthode de traitement du signal, l'ASS, et a défini à travers celle-ci des variables qui ont été suffisamment fidèles à l'évolution de l'état de l'outil et robustes vis-à-vis du bruit. Les résultats obtenus sont encourageants pour l'utilisation des techniques de Machine Learning dans le domaine de la surveillance d'usure d'outils. On devra, néanmoins, rappeler l'exigence de tenir compte du temps de traitement du signal si l'on veut implémenter un système in situ.

Cette étude a mis en évidence deux aspects importants : la forte richesse en information des composantes hautes fréquences des signaux vibratoires et la robustesse des indicateurs définis qui prouve la possibilité de s'affranchir du bruit inutile par la combinaison de l'ASS et d'un filtrage passe-bande.

Remerciements. Les auteurs remercient la société M.P. Colinet sprl pour sa diligente collaboration et la Coopération Technique Belge pour son appui financier.

\section{Références}

[1] E. Jantunen, Indirect multisignal monitoring and diagnosis of drill wear, Ph.D. Thesis, Helsinki University of technology, 2006

[2] C.S. Lee, D.A. Dornfeld, Design and implementation of sensor-based tool-wear monitoring systems, Mechanical Systems and Signal Processing 10 (1996) 328-347 
[3] H.V. Ravindra, Y.G. Srinisvasa, R. Krishnamurthy, Modeling of tool wear based on cutting forces in turning, Wear 169 (1993) 25-32

[4] I. Abu-Mahfouz, Drilling wear detection and classification using vibration signals and artificial neural network, Int. J. Machine Tools Manufacture 43 (2003) 707-720

[5] T. Obikawa, J. Shinozuka, Monitoring of flank wear of coated tools in high speed machining with a neural network art2, Int. J. Machine Tools Manufacture 44 (2004) $1311-1318$

[6] Dimla E. Dimla Snr, Sensor signals for tool-wear monitoring in metal cutting operations, a review of methods, Int. J. Machine Tools Manufacture 40 (200) 1073-1098

[7] D.E. Dimla Snr, P.M. Lister, On-line metal cutting tool condition monitoring I: force and vibration analyses, Int. J. Machine Tools Manufacture 40 (2000) 739-768

[8] T.I. El-Wardany, D. Gao, M.A. Elbestawi, Tool condition monitoring in drilling using vibration signature analysis, Int. J. Machine Tools Manufacture 36 (1996) 687-711

[9] C.Y. Jiang, Y.Z. Zhang, H.J. Xu, In-process monitoring of tool wear stage by the frequency band-energy method, Annals of the CIRP 36 (1997) 45-48

[10] R.E. Haber, J.E. Jiménez, C.R. Peres, J.R. Alique, An investigation of tool-wear monitoring in a high-speed machining process, Sensors and Actuators A 16 (2004) 539545

[11] X. Li, S. Dong, P.K. Venuvinod, Hybrid learning for tool wear monitoring, Int. J. Adv. Manuacturing Techn. 16 (2000) 303-307

[12] D. Shi, N.N. Gindy, Tool wear predictive model based on least squares support vector machines, Mechanical Systems and Signal Processing (2006) doi :10.1016/j.ymssp.2006.07.016
[13] G. O’Donnel, P. Young, K. Kelly, G. Byrne, Towards the improvement of tool condition monitoring systems in the manufacturing environment, J. Mat. Processing Tech. 119 (2001) 133-139

[14] M. Ghil, M.R. Allen, M.D. Dettinger, K. Ide, D. Kondrashov, M.E. Mann, A.W. Robertson, A. Saunders, Y. Tian, F. Varadi, P. Yiou, Advanced spectral methods for climatic time series, Rev. Geophys. 40 (2002) 1.1-1.41

[15] D.R. Salgado, F.J. Alonso, Tool wear detection in turning operations using singular spectrum analysis, J. Materials Processing Tech. 171 (2006) 451-458

[16] C. Aldrich, M. Barkhuizen, Process system identification strategies based on the use of singular spectrum analysis, Minerals engineering 16 (2003) 815-826

[17] G.T. Jemwa, C. Aldrich, Classification of process dynamics with Monte Carlo singular spectrum analysis, Computers and Chemical Eng. 30 (2006) 816-831

[18] D.S. Broomhead, G. King, Extracting qualitative dynamics from experimental data, Phys. D 20 (1986) 217-236

[19] N. Golyandina, V. Nekrutkin, A. Zhigljavsky, Analysis of Time Series Structure- SSA and related techniques, Chapman \& Hall, CRC, 2001

[20] P. Yiou, D. Sornette, M. Ghil, Data-adaptive wavelets and multi-scale singular spectrum analysis, Phys. D 142 (200) 254-290

[21] I.H. Witten, E. Frank, Data mining: Practical machine learning tools and techniques, Morgan Kaufmann, San Francisco, 2005

[22] R. Kohavi, A study of cross-validation and bootstrap for accuracy estimation and model selection, in: Proceedings of the Fourteenth International Joint Conference on Artificial Intelligence, Morgan Kaufmann, 1995, pp. 1137-1143 